\title{
OSCILLATION CRITERIA FOR FIRST-ORDER FORCED NONLINEAR DIFFERENCE EQUATIONS
}

\author{
RAVI P. AGARWAL, SAID R. GRACE, AND TIM SMITH
}

Received 11 August 2005; Revised 20 March 2006; Accepted 25 April 2006

Some new criteria for the oscillation of first-order forced nonlinear difference equations of the form $\Delta x(n)+q_{1}(n) x^{\mu}(n+1)=q_{2}(n) x^{\lambda}(n+1)+e(n)$, where $\lambda, \mu$ are the ratios of positive odd integers $0<\mu<1$ and $\lambda>1$, are established.

Copyright (c) 2006 Ravi P. Agarwal et al. This is an open access article distributed under the Creative Commons Attribution License, which permits unrestricted use, distribution, and reproduction in any medium, provided the original work is properly cited.

\section{Introduction}

We consider first-order forced nonlinear difference equations of the type

$$
\begin{gathered}
\Delta x(n)+p(n) x(n+1)+q_{1}(n) x^{\mu}(n+1)=e(n), \\
\Delta x(n)=q_{2}(n) x^{\lambda}(n+1)+e(n), \\
\Delta x(n)+q_{1}(n) x^{\mu}(n+1)=q_{2}(n) x^{\lambda}(n+1)+e(n),
\end{gathered}
$$

where

(i) $\{p(n)\},\{e(n)\}$ are sequences of real numbers;

(ii) $\left\{q_{i}(n)\right\}, i=1,2$, are sequences of positive real numbers;

(iii) $\lambda, \mu$ are ratios of positive odd integers with $0<\mu<1$ and $\lambda>1$.

By a solution of equation $(1, i), i=1,2,3$, we mean a nontrivial sequence $\{x(n)\}$ which is defined for $n \geq n_{0} \in \mathbb{N}=\{0,1,2, \ldots\}$ and satisfies equation $(1, i), i=1,2,3$, and $n=$ $1,2, \ldots$ A solution $\{x(n)\}$ of any of the equations $(1, i), i=1,2,3$, is said to be oscillatory if for every $n_{1} \in \mathbb{N}, n_{1}>0$, there exists an $n \geq n_{1}$ such that $x(n) x(n+1) \leq 0$, otherwise, it is nonoscillatory. Any of the equations $(1, i), i=1,2,3$, is said to be oscillatory if all its solutions are oscillatory.

In recent years, there has been an increasing interest in studying the oscillation and nonoscillation of solutions of difference equations. For example, see $[1,3-5]$ and the 


\section{Discrete first-order forced oscillation}

references cited therein. It is known that (1.1) and (1.2) with $e(n) \equiv p(n) \equiv 0$ are oscillatory if

$$
\sum^{\infty} q_{1}(n)=\infty, \quad \sum^{\infty} q_{2}(n)=\infty
$$

respectively. These conditions are also sufficient for the oscillation of (1.1) and (1.2) with $p(n) \equiv 0$ provided that there exists an oscillatory sequence $\{\eta(n)\}$ such that $\Delta \eta(n)=e(n)$.

In this paper, we are interested to establish some new criteria for the oscillation of (1.1)-(1.3) without imposing the above restriction on $\{e(n)\}$. In Section 2, we present some sufficient conditions for the oscillation of (1.1) and employ the same techniques to obtain oscillation results for the neutral equation

$$
\Delta(x(n)-c(n) x[n-\tau])+p(n) x(n+1)+q_{1}(n) x^{\mu}[n-\tau+1]=e(n),
$$

where $\{c(n)\}$ is a sequence of nonnegative real numbers and $\tau$ is any real number. In Section 3, we investigate the oscillatory property of (1.2) and discuss the case when $\lambda=1$, that is, the linear case. Section 4 is devoted to the study of the oscillatory behavior of (1.3). We also proceed further in this direction and obtain oscillation criteria for second-order equations of the form

$$
\Delta^{2} x(n-1)+q_{1}(n) x^{\mu}(n)=q_{2}(n) x^{\lambda}(n+1)+e(n)
$$

here $\lambda$ can assume the value 1 .

We note that the results of this paper are presented in a form which is essentially new and of high degree of generality. Also, for related results in oscillation of forced differential equations, we refer to our earlier paper [2].

\section{Oscillation criteria for (1.1)}

In order to discuss our results, we need the following lemma.

LEMMA 2.1 [6]. If $A$ and $B$ are nonnegative, then

(i) $A^{\lambda}-\lambda A B^{\lambda-1}-(1-\lambda) B^{\lambda} \geq 0, \lambda>1$;

(ii) $A^{\mu}-\mu A B^{\mu-1}-(1-\mu) B^{\mu} \leq 0,0<\mu<1$. Note that equality holds if and only if $A=B$.

The following theorem provides sufficient conditions for the oscillation of (1.1).

Theorem 2.2. Let $\{H(m, n): m, n \in \mathbb{N}, m \geq n \geq 0\}$ be a double sequence satisfying

$$
\begin{aligned}
H(m, m) & =0 \quad \text { for } m \geq 0, \quad H(m, n)>0 \quad \text { for } m>n \geq 0 \\
h(m, n) & =H(m, n)-H(m, n+1)>0 \quad \text { for } m>n \geq 0 \\
-P(m, n) & =h(m, n)+p(n) H(m, n)<0 \quad \text { for } m>n \geq 0 .
\end{aligned}
$$


Ravi P. Agarwal et al. 3

If, for $k \geq n_{0} \in \mathbb{N}$,

$$
\begin{aligned}
& \limsup _{m \rightarrow \infty} \frac{1}{H(m, k)} \sum_{n=k}^{m-1}[H(m, n) e(n)-Q(m, n)]=+\infty, \\
& \liminf _{m \rightarrow \infty} \frac{1}{H(m, k)} \sum_{n=k}^{m-1}[H(m, n) e(n)-Q(m, n)]=-\infty,
\end{aligned}
$$

where

$$
Q(m, n)=(1-\mu) \mu^{\mu /(1-\mu)}\left(\frac{P^{\mu}(m, n)}{H(m, n)}\right)^{1 /(\mu-1)} q_{1}^{1 /(1-\mu)}(n)
$$

then (1.1) is oscillatory.

Proof. Let $\{x(n)\}$ be an eventually positive solution of (1.1). Multiplying (1.1) by $H(m, n)$ for $m>n \geq k \in \mathbb{N}$, summing from $k$ to $m$, and using (2.1), we obtain

$$
\begin{aligned}
\sum_{n=k}^{m} H(m, n) e(n)= & \sum_{n=k}^{m} H(m, n) \Delta x(n)+\sum_{n=k}^{m} H(m, n) p(n) x(n+1) \\
& +\sum_{n=k}^{m} H(m, n) q_{1}(n) x^{\mu}(n+1) \\
= & -H(m, k) x(k)+\sum_{n=k}^{m-1} h(m, n) x(n+1)+\sum_{n=k}^{m} H(m, n) p(n) x(n+1) \\
& +\sum_{n=k}^{m} H(m, n) q_{1}(n) x^{\mu}(n+1) .
\end{aligned}
$$

Now,

$$
\begin{aligned}
& \frac{1}{H(m, k)} \sum_{n=k}^{m-1} H(m, n) e(n) \\
& \quad=-x(k)+\frac{1}{H(m, k)} \sum_{n=k}^{m-1}\left[H(m, n) q_{1}(n) x^{\mu}(n+1)-P(m, n) x(n+1)\right] .
\end{aligned}
$$

Set

$$
\begin{aligned}
& A=\left[H(m, n) q_{1}(n)\right]^{1 / \mu} x(n+1), \\
& B=\left(\frac{1}{\mu} P(m, n)\left(H(m, n) q_{1}(n)\right)^{-1 / \mu}\right)^{1 /(\mu-1)}
\end{aligned}
$$


4 Discrete first-order forced oscillation

and apply Lemma 2.1(ii) in (2.8) to obtain

$$
\begin{aligned}
& \frac{1}{H(m, k)} \sum_{n=k}^{m-1}\left[H(m, n) e(n)-(1-\mu)\left(\mu^{\mu /(1-\mu)}\right) \times P^{\mu /(1-\mu)}(m, n)\left(H(m, n) q_{1}(n)\right)^{1 /(1-\mu)}\right] \\
& \quad \leq-x(k) .
\end{aligned}
$$

Taking limsup as $m \rightarrow \infty$ in the above inequality, we obtain a contradiction to condition (2.4). If $\{x(n)\}$ is eventually negative, then reasoning as above leads to a contradiction with the condition (2.5). This completes the proof.

The following corollary is immediate.

Corollary 2.3. Let the sequence $\{H(m, n)\}$ be as in Theorem 2.2 such that (2.1)-(2.3) hold. If, for $k \geq n_{0} \in \mathbb{N}$,

$$
\begin{aligned}
& \limsup _{m \rightarrow \infty} \frac{1}{H(m, k)} \sum_{n=k}^{m-1} H(m, n) e(n)=+\infty, \\
& \liminf _{m \rightarrow \infty} \frac{1}{H(m, k)} \sum_{n=k}^{m-1} H(m, n) e(n)=-\infty, \\
& \lim _{m \rightarrow \infty} \frac{1}{H(m, k)} \sum_{n=k}^{m}\left(\frac{P^{\mu}(m, n)}{H(m, n)}\right)^{1 /(\mu-1)} q_{1}^{1 /(\mu-1)}(n)<\infty,
\end{aligned}
$$

then (1.1) is oscillatory.

Remark 2.4. It is easy to see that conditions (2.4) and (2.5) may be replaced by

$$
\begin{aligned}
& \limsup _{m \rightarrow \infty} \frac{1}{H(m, k)} \sum_{n=k}^{m-1}[H(m, n) e(n)-Q(m, n)]>0, \\
& \liminf _{m \rightarrow \infty} \frac{1}{H(m, k)} \sum_{n=k}^{m-1}[H(m, n) e(n)-Q(m, n)]<0,
\end{aligned}
$$

respectively.

Remark 2.5. Theorem 2.2 remains valid if $p(n) \equiv 0$ and $q_{1}(n)$ is of variable sign, that is, $q_{1}(n)$ takes the form

(ii) ${ }^{\prime} q_{1}(n)=a(n)-b(n), n \geq n_{0} \in \mathbb{N}$, where $\{a(n)\}$ and $\{b(n)\}$ are sequences of positive real numbers.

In this case, we have the following result for the equation

$$
\Delta x(n)+q_{1}(n) x^{\mu}(n+1)=e(n) .
$$

Theorem 2.6. Let $\{H(m, n)\}$ be defined as in Theorem 2.2 satisfying conditions (2.1) and (2.2). If conditions (2.4) and (2.5) hold with $Q(m, n)$ defined in Theorem 2.2 with $q_{1}(n)$ replaced by $b(n)$, then (1.1)' is oscillatory. 
Proof. Let $\{x(n)\}$ be an eventually positive solution of $(1.1)^{\prime}$. As in the proof of Theorem 2.2, we obtain (2.8). Now

$$
\begin{aligned}
& \frac{1}{H(m, k)} \sum_{n=k}^{m-1} H(m, n) e(n) \\
& \quad \geq-x(k)+\frac{1}{H(m, k)} \sum_{n=k}^{m-1}\left[h(m, n) x(n+1)-H(m, n) b(n) x^{\mu}(n+1)\right] .
\end{aligned}
$$

Proceeding as in the proof of Theorem 2.2, taking liminf of both sides of the resulting inequality as $m \rightarrow \infty$, and using condition (2.5), we arrive at the desired contradiction.

Corollary 2.7. Let $-P(n):=1+p(n)<0$ for $n \geq k \in \mathbb{N}$. If

$$
\begin{aligned}
& \limsup _{n \rightarrow \infty}\left[e(n)-(1-\mu) \mu^{\mu /(1-\mu)} P^{\mu /(\mu-1)}(n) q_{1}^{1 /(1-\mu)}(n)\right]>0, \\
& \liminf _{n \rightarrow \infty}\left[e(n)-(1-\mu) \mu^{\mu /(1-\mu)} P^{\mu /(\mu-1)}(n) q_{1}^{1 /(1-\mu)}(n)\right]<0,
\end{aligned}
$$

then (1.1) is oscillatory.

Proof. It suffices to note that in Theorem 2.2 for the choice $H(m, n)=H(n+1, n)>0$, $m>n \geq 0$,

$$
\begin{gathered}
h(m, n)=H(n+1, n)-H(n+1, n+1)=H(n+1, n)>0 \quad \text { for } m>n \geq 0, \\
-P(m, n)=-P(n) H(n+1, n)<0 \quad \text { for } m>n \geq 0,
\end{gathered}
$$

and for $k=n=m-1$,

$$
Q(m, n)=(1-\mu) \mu^{\mu /(1-\mu)}\left(P^{\mu /(\mu-1)}(m-1) H(m, m-1)\right) q_{1}^{1 /(1-\mu)}(m-1),
$$

so that the assumptions (2.4) and (2.5) are reduced to (2.14).

The following result is concerned with the oscillatory behavior of all bounded solutions of the equation

$$
\Delta x(n)+p(n) x(n)+q_{1}(n) x^{\mu}(n)=e(n),
$$

where $p(n), q_{1}(n), e(n)$, and $\mu$ are as in (1.1).

Theorem 2.8. Let $p(n)<1$ for $n \geq k \in \mathbb{N}$. If both

$$
\begin{aligned}
& \limsup _{n \rightarrow \infty}\left[e(n)-(1-\mu) \mu^{\mu /(1-\mu)}(1-p(n))^{\mu /(\mu-1)} q_{1}^{1 /(1-\mu)}(n)\right]=+\infty, \\
& \liminf _{n \rightarrow \infty}\left[e(n)-(1-\mu) \mu^{\mu /(1-\mu)}(1-p(n))^{\mu /(\mu-1)} q_{1}^{1 /(1-\mu)}(n)\right]=-\infty,
\end{aligned}
$$

then all bounded solutions of (2.17) are oscillatory. 
Proof. Let $\{x(n)\}$ be an eventually positive and bounded solution of (1.1). From (2.17) it follows that

$$
x(n+1)-(1-p(n)) x(n)+q_{1}(n) x^{\mu}(n)=e(n) .
$$

Set

$$
A=q_{1}^{1 / \mu}(n) x(n), \quad B=\left(\frac{1}{\mu}(1-p(n)) q_{1}^{-1 / \mu}(n)\right)^{1 /(\mu-1)}
$$

and apply Lemma 2.1(ii) in (2.20) to obtain

$$
\infty>x(n+1) \geq e(n)-(1-\mu) \mu^{\mu /(1-\mu)}(1-p(n))^{\mu /(1-\mu)} q_{1}^{1 /(1-\mu)}(n), \quad n \geq k .
$$

Taking limsup as $n \rightarrow \infty$ in the above inequality, we obtain a contradiction to condition (2.18). If $\{x(n)\}$ is eventually negative, then reasoning as above leads to a contradiction with the condition (2.19). This completes the proof.

The following examples are illustrative.

Example 2.9. Consider the forced difference equation

$$
\Delta x(n)-2 x(n+1)+x^{1 / 3}(n+1)=(-1)^{(n+1) / 3}, \quad n \geq 0 .
$$

All conditions of Corollary 2.7 are satisfied and hence $(2.23)$ is oscillatory. One such solution is $x(n)=(-1)^{n}$.

Example 2.10. The forced difference equation

$$
\Delta x(n)-2 x(n)+n x^{1 / 3}(n)=n(-1)^{n / 3}-4(-1)^{n}, \quad n \geq 0,
$$

has a bounded oscillatory solution $x(n)=(-1)^{n}$. All conditions of Theorem 2.8 are satisfied.

Remark 2.11. We may note that Theorem 2.2 and Corollary 2.7 fail to apply to (1.1) with $p(n) \equiv 0$, while Theorem 2.8 is applicable to (2.17) with $p(n) \equiv 0, n \geq n_{0} \in \mathbb{N}$. In the former case, Theorem 2.8 takes the form of the following corollary.

Corollary 2.12. If

$$
\begin{aligned}
& \limsup _{n \rightarrow \infty}\left[e(n)-(1-\mu)\left(\mu^{\mu} q_{1}(n)\right)^{1 /(1-\mu)}\right]=\infty, \\
& \liminf _{n \rightarrow \infty}\left[e(n)-(1-\mu)\left(\mu^{\mu} q_{1}(n)\right)^{1 /(1-\mu)}\right]=-\infty,
\end{aligned}
$$

then all bounded solutions of (2.17) are oscillatory.

Next, we will apply the technique employed to present oscillation result for the neutral forced nonlinear difference equation of the form

$$
\Delta(x(n)-c(n) x[n-\tau])+p(n) x(n+1)+q_{1}(n) x^{\mu}[n-\tau+1]=e(n),
$$


where $p(n), q_{1}(n), e(n)$, and $\mu$ are as in (1.1), $\{c(n)\}$ is a sequence of nonnegative real numbers, and $\tau$ is any real number.

Theorem 2.13. Let the sequence $\{H(m, n)\}$ be as in Theorem 2.2 such that (2.1)-(2.3) hold and $h(m, n)+p(n) H(m, n) \leq 0$ for $m \geq n \geq k-\tau \in \mathbb{N}$. If

$$
\begin{aligned}
\limsup _{m \rightarrow \infty} \frac{1}{H(m, k)} \sum_{n=k}^{m-1}[H(m, n) e(n)- & (1-\mu) \mu^{\mu /(1-\mu)}(c(n+1) h(m, n))^{\mu /(\mu-1)} \\
& \left.\times\left(H(m, n) q_{1}(n)\right)^{1 /(1-\mu)}\right]=+\infty, \\
\liminf _{m \rightarrow \infty} \frac{1}{H(m, k)} \sum_{n=k}^{m-1}[H(m, n) e(n)- & (1-\mu) \mu^{\mu /(1-\mu)}(c(n+1) h(m, n))^{\mu /(\mu-1)} \\
& \left.\times\left(H(m, n) q_{1}(n)\right)^{1 /(1-\mu)}\right]=-\infty,
\end{aligned}
$$

then (2.26) is oscillatory.

Proof. Let $\{x(n)\}$ be an eventually positive solution of (2.26). Multiplying the (2.26) by $H(m, n)$ for $m>n \geq k-\tau \in \mathbb{N}$, summing from $k$ to $m$, and using (2.1), we have

$$
\begin{aligned}
\sum_{n=k}^{m} H(m, n) e(n)= & -H(m, k)(x(k)-c(k) x[k-\tau])+\sum_{n=k}^{m-1}(h(m, n)+p(n) H(m, n)) x(n+1) \\
& +\sum_{n=k}^{m-1}\left(H(m, n) q_{1}(n) x^{\mu}[n-\tau+1]-h(m, n) c(n+1) x[n-\tau+1]\right),
\end{aligned}
$$

or

$$
\begin{aligned}
& \frac{1}{H(m, k)} \sum_{n=k}^{m-1} H(m, n) e(n) \\
& \leq-(x(k)-c(k) x[k-\tau]) \\
& \quad+\frac{1}{H(m, k)} \times \sum_{n=k}^{m-1}\left(H(m, n) q_{1}(n) x^{\mu}[n-\tau+1]-h(m, n) c(n+1) x[n-\tau+1]\right) .
\end{aligned}
$$

Set

$$
\begin{aligned}
& A=\left(H(m, n) q_{1}(n)\right)^{1 / \mu} x[n-\tau+1], \\
& B=\left(\frac{1}{\mu} h(m, n) c(n+1)\left(H(m, n) q_{1}(n)\right)^{-1 / \mu}\right)^{1 /(\mu-1)}
\end{aligned}
$$


8 Discrete first-order forced oscillation

and apply Lemma 2.1(ii) in (2.29) to obtain

$$
\begin{aligned}
& \frac{1}{H(m, k)} \sum_{n=k}^{m-1}\left[H(m, n) e(n)-(1-\mu) \mu^{\mu /(1-\mu)}(h(m, n) c(n+1))^{\mu /(\mu-1)}\left(H(m, n) q_{1}(n)\right)^{1 /(1-\mu)}\right] \\
& \quad \leq c(k) x[k-\tau]-x(k) .
\end{aligned}
$$

The rest of the proof is similar to that of Theorem 2.2 and hence omitted.

\section{Oscillation of (1.2)}

Our main oscillation criterion for (1.2) is the following result.

Theorem 3.1. Let the sequences $\{H(m, n)\}$ be as in Theorem 2.2 such that (2.1) and (2.2) hold. If for $k \geq n_{0} \in \mathbb{N}$,

$$
\begin{aligned}
& \limsup _{m \rightarrow \infty} \frac{1}{H(m, k)} \sum_{k=n}^{m}[H(m, n) e(n)-C(m, n)]=+\infty, \\
& \liminf _{m \rightarrow \infty} \frac{1}{H(m, k)} \sum_{k=n}^{m}[H(m, n) e(n)-C(m, n)]=-\infty,
\end{aligned}
$$

where

$$
C(m, n)=(\lambda-1) \lambda^{\lambda /(1-\lambda)}\left(\frac{h^{\lambda}(m, n)}{H(m, n)}\right)^{1 /(\lambda-1)} q_{2}^{1 /(1-\lambda)}(n), \quad m>n \geq k
$$

then (1.2) is oscillatory.

Proof. Let $\{x(n)\}$ be an eventually positive solution of (1.2). Multiplying (1.2) by $H(m, n)$ for $m>n \geq k \geq n_{0} \in \mathbb{N}$, summing from $k$ to $m$, and using (2.1), we have

$$
\begin{aligned}
\sum_{n=k}^{m} H(m, n) e(n) & =\sum_{n=k}^{m} H(m, n) \Delta x(n)-\sum_{n=k}^{m} H(m, n) q_{2}(n) x^{\lambda}(n+1) \\
& =-H(m, k) x(k)+\sum_{n=k}^{m-1} h(m, n) x(n+1)-\sum_{n=k}^{m} H(m, n) q_{2}(n) x^{\lambda}(n+1),
\end{aligned}
$$

or

$$
\begin{aligned}
& \frac{1}{H(m, k)} \sum_{n=k}^{m-1} H(m, n) e(n) \\
& \quad=-x(k)+\frac{1}{H(m, n)} \sum_{n=k}^{m-1}\left[h(m, n) x(n+1)-H(m, n) q_{2}(n) x^{\lambda}(n+1)\right]
\end{aligned}
$$


Set

$$
A=\left(H(m, n) q_{2}(n)\right)^{1 / \lambda} x(n+1), \quad B=\left(\frac{1}{\lambda} h(m, n)\left(H(m, n) q_{2}(n)\right)^{-1 / \lambda}\right)^{1 /(\lambda-1)}
$$

and apply Lemma 2.1(i) in (3.4) to get

$$
\begin{aligned}
& \frac{1}{H(m, k)} \sum_{n=k}^{m-1} H(m, n) e(n) \\
& \quad \leq-x(k)+\frac{1}{H(m, k)} \sum_{n=k}^{m-1}(\lambda-1) \lambda^{\lambda /(1-\lambda)} h^{\lambda /(\lambda-1)}(m, n)\left(H(m, n) q_{2}(n)\right)^{1 /(1-\lambda)} .
\end{aligned}
$$

The rest of the proof is similar to that of Theorem 2.2 and hence omitted.

Corollary 3.2. If

$$
\begin{gathered}
\limsup _{n \rightarrow \infty}\left[e(n)-(\lambda-1) \lambda^{\lambda /(1-\lambda)} q_{2}^{1 /(1-\lambda)}(n)\right]>0, \\
\liminf _{n \rightarrow \infty}\left[e(n)-(\lambda-1) \lambda^{\lambda /(1-\lambda)} q_{2}^{1 /(1-\lambda)}(n)\right]<0,
\end{gathered}
$$

then (1.2) is oscillatory.

The following example is illustrative.

Example 3.3. The superlinear forced difference equation

$$
\Delta x(n)=\frac{1}{(n+1)^{2}} x^{3}(n+1)+(-1)^{n+1} n
$$

has an oscillatory solution $x(n)=(-1)^{n} n$. All conditions of Corollary 3.2 are satisfied.

Remark 3.4. When $\lambda=1$, we see that the inequality (3.4) in the proof of Theorem 3.1 reduces to

$$
\frac{1}{H(m, k)} \sum_{n=k}^{m-1} H(m, k) e(n) \leq-x(k)-\frac{1}{H(m, k)} \sum_{n=k}^{m-1}\left[H(m, n) q_{2}(n)-h(m, n)\right] x(n+1)
$$

and thus we obtain the following result.

Theorem 3.5. Let the sequence $\{H(m, n)\}$ be as in Theorem 2.2 such that the conditions (2.1) and (2.2) hold, and

$$
H(m, n) q_{2}(n)-h(m, n) \geq 0 \quad \text { for } m>n \geq k
$$


If

$$
\begin{aligned}
& \limsup _{m \rightarrow \infty} \frac{1}{H(m, k)} \sum_{n=k}^{m} H(m, n) e(n)=+\infty, \\
& \liminf _{m \rightarrow \infty} \frac{1}{H(m, k)} \sum_{n=k}^{m} H(m, n) e(n)=-\infty,
\end{aligned}
$$

then (1.2) with $\lambda=1$ is oscillatory.

The following example dwells upon the importance of the above result.

Example 3.6. The linear forced difference equation

$$
\Delta x(n)=x(n+1)+(-1)^{n+1} n
$$

has an oscillatory solution $x(n)=(-1)^{n} n$. All conditions of Theorem 3.5 with $H(m, n)=$ $H(n+1, n)>0, m>n \geq 0$, and for $k=n=m-1$, are fulfilled.

\section{Oscillation of (1.3)}

We will combine some of our results in Sections 2 and 3 to obtain oscillation criteria for (1.3).

Theorem 4.1. Let the sequence $\{H(m, n)\}$ be as in Theorem 2.2 such that conditions (2.1) and (2.2) hold. If there exists a constant $\alpha>0$ such that for $k \in \mathbb{N}$,

$$
\begin{aligned}
& \limsup _{m \rightarrow \infty} \frac{1}{H(m, k)} \sum_{n=k}^{m-1}[H(m, n) e(n)-B(m, n)]=+\infty, \\
& \liminf _{m \rightarrow \infty} \frac{1}{H(m, k)} \sum_{n=k}^{m-1}[H(m, n) e(n)-B(m, n)]=-\infty,
\end{aligned}
$$

where

$$
\begin{aligned}
B(m, n)= & (1-\mu)\left(\frac{\mu}{\alpha}\right)^{\mu /(1-\mu)}\left(\frac{h^{\mu}(m, n)}{H(m, n)}\right)^{1 /(\mu-1)} q_{1}^{1 /(1-\mu)}(n) \\
& +(\lambda-1)\left(\frac{\lambda}{\alpha+1}\right)^{\lambda /(1-\lambda)}\left(\frac{h^{\lambda}(m, n)}{H(m, n)}\right)^{1 /(\lambda-1)} q_{2}^{1 /(1-\lambda)}(n),
\end{aligned}
$$

then (1.3) is oscillatory. 
Proof. Let $\{x(n)\}$ be an eventually positive solution of (1.3). Multiplying (1.3) by $H(m, n)$, summing from $k$ to $m \geq k$, and using condition (2.1), we get

$$
\begin{aligned}
\sum_{n=k}^{m} H(m, n) e(n)= & \sum_{n=k}^{m} H(m, n) \Delta x(n)+\sum_{n=k}^{m} H(m, n) q_{1}(n) x^{\mu}(n+1) \\
& -\sum_{n=k}^{m} H(m, n) q_{2}(n) x^{\lambda}(n+1) \\
= & -H(m, k) x(k)+\sum_{n=k}^{m-1} h(m, n) x(n+1)+\sum_{n=k}^{m-1} H(m, n) q_{1}(n) x^{\mu}(n+1) \\
& -\sum_{n=k}^{m-1} H(m, n) q_{2}(n) x^{\lambda}(n+1) \\
= & -H(m, k) x(k)+\sum_{n=k}^{m-1}\left[H(m, n) q_{1}(n) x^{\mu}(n+1)-\alpha h(m, n) x(n+1)\right] \\
& +\sum_{n=k}^{m-1}\left[(\alpha+1) h(m, n) x(n+1)-H(m, n) q_{2}(n) x^{\lambda}(n+1)\right] .
\end{aligned}
$$

As in the proof of Theorem 2.2 with $P(m, n)$ being replaced by $\alpha h(m, n)$ and Theorem 3.1 with $h(m, n)$ being replaced by $(\alpha+1) h(m, n)$, we see that

$$
\begin{aligned}
\frac{1}{H(m, k)} \sum_{n=k}^{m-1} H(m, n) e(n) & \\
\leq-x(k)+\frac{1}{H(m, k)} \sum_{n=k}^{m-1}[ & (1-\mu)\left(\frac{\mu}{\alpha}\right)^{\mu /(1-\mu)} h^{\mu /(\mu-1)}(m, n)\left(H(m, n) q_{1}(n)\right)^{1 /(1-\mu)} \\
& \left.+(\lambda-1)\left(\frac{\lambda}{\alpha+1}\right)^{\lambda /(1-\lambda)} h^{\lambda /(\lambda-1)}(m, n)\left(H(m, n) q_{2}(n)\right)^{1 /(1-\lambda)}\right] .
\end{aligned}
$$

The rest of the proof is similar to that of Theorem 2.2 and hence omitted.

COROLlary 4.2. If there exists a positive constant $\alpha$ such that

$$
\begin{aligned}
& \limsup _{n \rightarrow \infty}\left[e(n)-(1-\mu)\left(\frac{\mu}{\alpha}\right)^{\mu /(1-\mu)} q_{1}^{1 /(1-\mu)}(n)-(\lambda-1)\left(\frac{\lambda}{\alpha+1}\right)^{\lambda /(\lambda-1)} q_{2}^{1 /(1-\lambda)}(n)\right]>0, \\
& \liminf _{n \rightarrow \infty}\left[e(n)-(1-\mu)\left(\frac{\mu}{\alpha}\right)^{\mu /(1-\mu)} q_{1}^{1 /(1-\mu)}(n)-(\lambda-1)\left(\frac{\lambda}{\alpha+1}\right)^{\lambda /(\lambda-1)} q_{2}^{1 /(1-\lambda)}(n)\right]<0,
\end{aligned}
$$

then (1.3) is oscillatory. 
The following example is illustrative.

Example 4.3. The forced nonlinear difference equation

$$
\Delta x(n)+\frac{1}{(n+1)^{1 / 3}} x^{1 / 3}(n+1)=\frac{1}{(n+1)^{3}} x^{3}(n+1)+(-1)^{n+1}(2 n+1)
$$

has an oscillatory solution $x(n)=(-1)^{n} n$. All conditions of Corollary 4.2 are satisfied with $\alpha=6$.

Now, we will apply the technique employed above to obtain oscillation results for second-order difference equations of the form

$$
\Delta^{2} x(n-1)+q_{1}(n) x^{\mu}(n)=q_{2}(n) x^{\lambda}(n+1)+e(n),
$$

where $q_{1}, q_{2}, e, \mu$, and $\lambda$ are as in (1.3).

Theorem 4.4. Let the sequence $\{H(m, n)\}$ be as in Theorem 2.2 such that conditions (2.1) and (2.2) hold. If, for $k \in \mathbb{N}$,

$$
\begin{aligned}
& \limsup _{m \rightarrow \infty} \frac{1}{H(m, k)} \sum_{n=k}^{m}[H(m, n) e(n)-D(m, n)]=+\infty, \\
& \liminf _{m \rightarrow \infty} \frac{1}{H(m, k)} \sum_{n=k}^{m}[H(m, n) e(n)-D(m, n)]=-\infty,
\end{aligned}
$$

where

$$
\begin{aligned}
D(m, n)= & (1-\mu) \mu^{\mu /(1-\mu)}\left(\frac{h^{\mu}(m, n)}{H(m, n)}\right)^{1 /(\mu-1)} q_{1}^{1 /(1-\mu)}(n) \\
& +(\lambda-1) \lambda^{\lambda /(1-\lambda)}\left(\frac{h^{\lambda}(m, n)}{H(m, n)}\right)^{1 /(\lambda-1)} q_{2}^{1 /(1-\lambda)}(n),
\end{aligned}
$$

then (4.7) is oscillatory.

Proof. Let $\{x(n)\}$ be an eventually positive solution of (4.7). Multiplying (4.7) by $H(m, n)$, summing from $k$ to $m$, and using (2.1), we obtain

$$
\begin{aligned}
\sum_{n=k}^{m} H(m, n) e(n)= & \sum_{n=k}^{m} H(m, n) \Delta^{2} x(n-1) \\
& +\sum_{n=k}^{m} H(m, n) q_{1}(n) x^{\mu}(n)-\sum_{n=k}^{m} H(m, n) q_{2}(n) x^{\lambda}(n+1) \\
= & -H(m, k) \Delta x(k-1)+\sum_{n=k}^{m-1} h(m, n) \Delta x(n)+\sum_{n=k}^{m} H(m, n) q_{1}(n) x^{\mu}(n) \\
& -\sum_{n=k}^{m} H(m, n) q_{2}(n) x^{\lambda}(n+1)
\end{aligned}
$$


or

$$
\begin{aligned}
\frac{1}{H(m, k)} \sum_{n=k}^{m} H(m, n) e(n)= & -\Delta x(k-1)+\frac{1}{H(m, k)} \sum_{n=k}^{m-1}\left[H(m, n) q_{1}(n) x^{\mu}(n)-h(m, n) x(n)\right] \\
& +\frac{1}{H(m, k)} \sum_{n=k}^{m-1}\left[h(m, n) x(n+1)-H(m, n) q_{2}(n) x^{\lambda}(n+1)\right] .
\end{aligned}
$$

Proceeding as in the proof of Theorem 4.1, we arrive at the desired contradiction.

Similarly, we present the following result for the equation

$$
\Delta^{2} x(n-1)+p(n) x(n)+q_{1}(n) x^{\mu}(n)=e(n)
$$

where $p, q_{1}, e$, and $\mu$ are as in (1.1).

Theorem 4.5. Let the sequence $\{H(m, n)\}$ be as in Theorem 2.2 such that conditions (2.1) and (2.2) hold, and

$$
h(m, n)+p(n) H(m, n) \leq 0 \quad \text { for } m>n \geq k .
$$

If conditions (4.8) hold with

$$
D(m, n)=(1-\mu) \mu^{\mu /(1-\mu)}\left(\frac{h^{\mu}(m, n)}{H(m, n)}\right)^{1 /(\mu-1)} q_{1}^{1 /(1-\mu)}(n),
$$

then (4.12) with is oscillatory.

Proof. Let $\{x(n)\}$ be an eventually positive solution of (4.12). As in the proof of Theorem 4.4 , we have

$$
\begin{aligned}
\sum_{n=k}^{m} H(m, n) e(n)= & -H(m, k) \Delta x(k-1)+\sum_{n=k}^{m-1}(H(m, n) p(n)+h(m, n)) x(n+1) \\
& +\sum_{n=k}^{m-1}\left[H(m, n) q_{1}(n) x^{\mu}(n)-h(m, n) x(n)\right] \\
\leq & -H(m, k) \Delta x(k-1)+\sum_{n=k}^{m-1}\left[H(m, n) q_{1}(n) x^{\mu}(n)-h(m, n) x(n)\right] .
\end{aligned}
$$

The rest of the proof is similar to that of Theorem 2.2 and hence omitted.

Also, we discuss the oscillatory behavior of a special case of (4.7), namely, the equation

$$
\Delta^{2} x(n-1)=q_{2}(n) x^{\lambda}(n+1)+e(n)
$$

here $\lambda$ can assume the value 1 , that is, the equation can be linear. 
14 Discrete first-order forced oscillation

Theorem 4.6. Let the sequences $\{H(m, n)\}$ be as in Theorem 2.2 such that conditions (2.1) and (2.2) hold. If conditions (4.8) hold with

$$
D(m, n)=(\lambda-1) \lambda^{\lambda /(1-\lambda)}\left(\frac{h^{\lambda}(m, n)}{H(m, n)}\right)^{1 /(\lambda-1)} q_{2}^{1 /(1-\lambda)}(n)
$$

then (4.16) with $\lambda>1$ is oscillatory.

Proof. Let $\{x(n)\}$ be an eventually positive solution of (4.16). As in the proof of Theorem 4.4 , one can easily find

$$
\sum_{n=k}^{m} H(m, n) e(n) \leq-H(m, k) \Delta x(k-1)+\sum_{n=k}^{m-1}\left[h(m, n) x(n+1)-H(m, n) q_{2}(n) x^{\lambda}(n+1)\right] .
$$

The rest of the proof is similar to that of Theorem 3.1 and hence omitted.

Remark 4.7. If we let $\lambda=1$ in (4.16), we see that the inequality (4.18) in the proof of Theorem 4.8 reduces to

$$
\begin{aligned}
& \frac{1}{H(m, k)} \sum_{n=k}^{m-1} H(m, n) e(n) \\
& \quad \leq-\Delta x(k-1)+\frac{1}{H(m, k)} \sum_{n=k}^{m-1}\left[h(m, n) x(n+1)-H(m, n) q_{2}(n) x(n+1)\right] .
\end{aligned}
$$

Thus, we obtain the following result.

Theorem 4.8. Let the sequence $\{H(m, n)\}$ be as in Theorem 2.2 such that conditions (2.1) and (2.2) hold, and

$$
H(m, n) q_{2}(n)-h(m, n) \geq 0 \quad \text { for } m \geq n \geq k .
$$

If

$$
\begin{aligned}
& \limsup _{m \rightarrow \infty} \sum_{n=k}^{m} H(m, n) e(n)=+\infty, \\
& \liminf _{m \rightarrow \infty} \sum_{n=k}^{m} H(m, n) e(n)=-\infty,
\end{aligned}
$$

then (4.16) with $\lambda=1$ is oscillatory.

For (4.12) with $p(n) \equiv 0$, we have the following result. 
THeOrem 4.9. If

$$
\begin{aligned}
& \limsup _{n \rightarrow \infty}\left[e(n)-(1-\mu)\left(\frac{\mu}{2}\right)^{\mu /(1-\mu)} q_{1}^{1 /(1-\mu)}(n)\right]=+\infty, \\
& \liminf _{n \rightarrow \infty}\left[e(n)-(1-\mu)\left(\frac{\mu}{2}\right)^{\mu /(1-\mu)} q_{1}^{1 /(1-\mu)}(n)\right]=-\infty
\end{aligned}
$$

then all bounded solutions of (4.12) with $p(n) \equiv 0$ are oscillatory.

Proof. Let $\{x(n)\}$ be an eventually positive and bounded solution of (4.12) with $p(n) \equiv 0$. Now,

$$
e(n)=(x(n+1)+x(n-1))+\left[q_{1}(n) x^{\mu}(n)-2 x(n)\right] .
$$

As in the proof of Theorem 2.6, we have

$$
e(n)-(1-\mu)\left(\frac{\mu}{2}\right)^{\mu /(1-\mu)} q_{1}^{1 /(1-\mu)}(n) \leq x(n+1)+x(n-1)<\infty .
$$

Taking limsup as $n \rightarrow \infty$, we obtain a contradiction to condition (4.22). This completes the proof.

Example 4.10. The forced second-order nonlinear difference equation

$$
\Delta^{2} x(n-1)+\frac{1}{\sqrt[3]{n}} x^{1 / 3}(n)=1
$$

has an unbounded solution $x(n)=n$. All conditions of Theorem 4.9 are satisfied except those on $e(n)$, that is, conditions (4.22) and (4.23).

Remark 4.11. We note that we can apply the technique presented here to obtain oscillation criteria for the neutral forced equations of type (2.26), that is,

$$
\Delta(x(n)-c(n) x[n-\tau])+q_{1}(n) x^{\mu}[n-\tau+1]=q_{2}(n) x^{\lambda}[n-\tau+1]+e(n),
$$

and also for forced neutral second-order nonlinear difference equations of the form

$$
\Delta^{2}(x(n)-c(n) x[n-\tau])+q_{1}(n) x^{\mu}[n-\tau+1]=q_{2}(n) x^{\lambda}[n-\tau+1]+e(n) .
$$

The formulations of these oscillations results and their proofs are left to the reader.

Remark 4.12. We note that the results of this paper are not applicable to unforced equations, that is, when $e(n) \equiv 0$.

\section{Acknowledgment}

The authors are grateful to the referees for their suggestions on the first draft of this paper. 


\section{References}

[1] R. P. Agarwal, M. Bohner, S. R. Grace, and D. O’Regan, Discrete Oscillation Theory, Hindawi, New York, 2005.

[2] R. P. Agarwal and S. R. Grace, Forced oscillation of nth-order nonlinear differential equations, Applied Mathematics Letters 13 (2000), no. 7, 53-57.

[3] R. P. Agarwal, S. R. Grace, and D. O'Regan, Oscillation Theory for Difference and Functional Differential Equations, Kluwer Academic, Dordrecht, 2000.

[4] R. P. Agarwal and P. J. Y. Wong, Advanced Topics in Difference Equations, Mathematics and Its Applications, vol. 404, Kluwer Academic, Dordrecht, 1997.

[5] M. Cecchi, Z. Došlá, and M. Marini, Nonoscillatory half-linear difference equations and recessive solutions, Advances in Difference Equations 2005 (2005), no. 2, 193-204.

[6] G. H. Hardy, J. E. Littlewood, and G. Pólya, Inequalities, 2nd ed., Cambridge Mathematical Library, Cambridge University Press, Cambridge, 1988.

Ravi P. Agarwal: Department of Mathematical Sciences, Florida Institute of Technology, Melbourne, FL 32901, USA

E-mail address: agarwal@fit.edu

Said R. Grace: Department of Engineering Mathematics, Faculty of Engineering, Cairo University, Orman, Giza 12221, Egypt

E-mail address: srgrace@eng.cu.edu.eg

Tim Smith: Department of Mathematical Sciences, Florida Institute of Technology, Melbourne, FL 32901, USA

E-mail address: smitht@fit.edu 